\title{
Facile Diabetic Retinopathy Detection using MRHE-FEED and Classification using Deep Convolutional Neural Network
}

\author{
Muhammad Zubair \\ Department of Electronics and Communication Engineering \\ Prasad V. Potluri Siddhartha Institute of Technology \\ Vijayawada, India \\ muhammadzub17@ieee.org
}

\author{
Umesh Kumar Naik M \\ Electronics and Communication Engineering \\ Prasad V. Potluri Siddhartha Institute of Technology \\ Vijayawada, India \\ umeshkumar.mudavath@gmail.com
}

\author{
Gunturi N V S Chandra Mouli \\ Department of Electrical and Electronics Engineering \\ Prasad V. Potluri Siddhartha Institute of Technology \\ Vijayawada, India \\ gunturicm@gmail.com
}

\begin{abstract}
Diabetic Retinopathy (DR) is an intricacy of diabetes that affects the eyes. In this paper, we have proposed a hybrid pre-processing and feature extraction technique named as Microaneurysm Retinal vein Haemorrhage Exudate (MRHE) extraction using Feature Enhancement and Edge Detection (FEED) which can extract all the features in a single step and with very less complexity. To classify the presence of $D R$, we have used an efficient Deep Convolutional Neural Network (D-CNN), model. The D-CNN model is trained with four salient features namely retinal veins, MA's, exudates, and haemorrhages which were extracted from the raw images using image-processing techniques. After training and testing the D-CNN model, we were able to classify the presence of DR based on the features extracted from the testing data. To implement this proposed method, we have used a dataset from the STructured Analysis of the Retina (STARE) Database, which comprises of retinal images taken under various imaging conditions using fundus photography. To demonstrate the legitimacy of the proposed method, we have compared our method with the existing DR detection and classification methods such as SVM, ANN,etc.. Performance evaluation results in terms of Accuracy and Recall show that the proposed algorithm outperforms other existing DR classification methods.
\end{abstract}

Index Terms-Diabetic Retinopathy (DR), Microaneurysm Retinal vein Haemorrhage Exudate (MRHE), Feature Enhancement and Edge Detection (FEED), Deep Convolution Neural Network (D-CNN)

\section{INTRODUCTION}

Diabetes is a metabolic syndrome that causes complications, including cardiovascular, nephritic, and eye disorders, due to which most of the research work is directed towards automated detection. It has been perceived as a leading global medical issue with the foreseen worldwide commonness of 350 million by the year 2030. An ascent in glucose levels related to diabetes is the known reason behind DR. DR advances through

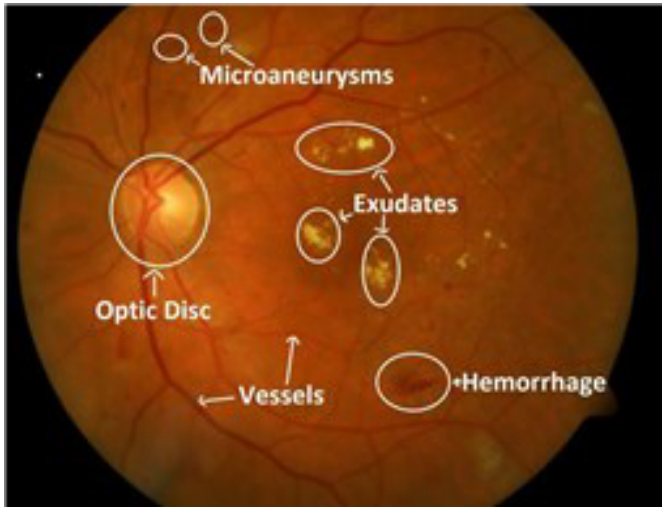

Fig. 1. Various features of retinal images in the human eye related to DR.

different levels. "The essential signs of DR incorporate MA's, little haemorrhages, exudates, and cotton wool spots, which happen because of impermeable and variation from the norm of blood dissemination in vessels. These initial syndromes are stated as non-proliferative DR (NPDR). There can even be prior signs of DR. In further advancement of disease blocked capillaries lead to the formation of new fragile vessels and retinal constriction, this phase is defined as Proliferative DR (PDR)." New veins turn up either close to the optic disc or somewhere else on the retinal surface. Since the ailment is treatable with deep observing and screening, evidently, it appears as though Automatic analysis and reviewing of retinal illnesses to developing need is an engineering solution. Various features of retinal images of the human eye related to DR are shown in Fig. 1.

Previously, numerous methods have been developed for 
the detection and classification of DR. These methods are based on the image processing and classification techniques including, Artificial Neural Network (ANN) [1], Singular Spectrum Analysis (SSA) [2], Morphological technique [3], Extracting Area and Number of MA [4], High Boost Filtering, Line Detectors, and OC-SVM [5]. In [6] they introduced a Feature Extraction and Classification of Retinal Images for Automated Detection of DR.

Although in the works mentioned above, various algorithms have been widely studied, the performance of the detection and classification of DR can be further improved by considering deep learning algorithms which can efficiently remove other artifacts.

The further sections of this paper are detailed and organized as follows: Section 2 presents methodology used in our work. Section 3 outlines the working of the proposed method to detect DR features and then classify them using D-CNN model. Section 4 illustrates the experimental results and observations that have been obtained. Section 5 discusses about the novelty of our proposed method with the existing methods. Finally, section 6 provides the conclusions and explains the potential future work that could be performed upon the proposal.

\section{Methodology}

\section{A. Morphological Opening (MO)}

MO eliminates small objects from a retinal fundus image without altering the shape and size of larger objects in the fundus image. The morphological opening function can be expressed as

$$
A^{\circ} B=(A \ominus B) \oplus B
$$

\section{B. Contrast Limited Adaptive Histogram Equalization (CLAHE)}

To overcome the over-amplification of the noise problems which exist is histogram equalization, we use CLAHE. CLAHE deals with the small regions of the retinal fundus image and computes several histograms, each compared to a particular area of the fundus image and used them to redistribute the brightness or contrast estimation of the image.

\section{Local Thresholding}

This technique is used to convert the image of the grayscale to pure black and white scale pixels. Unlike the other thresholding techniques used in image pre-processing, it chooses different threshold values for every pixel in the fundus image depending on the decomposition of neighbouring pixels.

The local threshold $\left(t h^{*}\right)$ is calculated as follows

$$
t h^{*}=\text { Argument } M A X\left(\sigma_{1}^{2} * \sigma_{2}^{2}\right)
$$

\section{Gaussian Blur}

This method is also known as Gaussian smoothing. It is most commonly used to reduce noise and detail in a retinal fundus image. For 2D images gaussian blur can be derived as shown in (3).

$$
G(a, b)=\left[\frac{1}{2 \pi \sigma^{2}}\right] \exp ^{-\frac{a^{2}+b^{2}}{2 \sigma^{2}}}
$$

\section{E. Laplacian of Gaussian ( $L O G$ )}

The Laplacian is a 2-D isotropic measure of the 2nd spatial derivative of a fundus image. It highlights regions of rapid intensity change which is very helpful in detecting the edges and enhances the contrast of the essential features and smoothens the other anomalous image features. LOG filter is derived by the partial differentiation of Gaussian function as given in (4) [7].

$$
\begin{gathered}
L O G=\Delta G_{\sigma}(a, b)=\frac{\partial^{2} G_{\sigma}(a, b)}{\partial a^{2}}+\frac{\partial^{2} G_{\sigma}(a, b)}{\partial b^{2}} \\
L O G=\left[\frac{a^{2}+b^{2}-2 \sigma^{2}}{\sigma^{4}}\right] \exp ^{-\frac{a^{2}+b^{2}}{2 \sigma^{2}}}
\end{gathered}
$$

Here we need to set some valid $\sigma$ value for different pathological cases.

\section{F. Sobel edge detection (SED)}

The name itself conveys to us that it is used to detect the edges. Sobel operator is a symmetrical gradient. Gradient compares to the main subordinate. For a continuous function $f 1(x, y)$, in the position $p 1(x, y)$, its gradient can be communicated as a vector (the two segments are two first subordinates which are along the $\mathrm{X}$ and $\mathrm{Y}$ course individually.

$$
\nabla f(x, y)=\left[G_{x} G_{y}\right]^{\top}=[\delta f / \partial x \partial f / \partial y]
$$

The principal methodology of the Sobel operator, $\mathrm{G}(\mathrm{x}, \mathrm{y})$ is to register the magnitude of the gradient at every pixel area utilizing the condition,

$$
G(\mathrm{x}, \mathrm{y})=\sqrt{\mathrm{G}_{\mathrm{x}}^{2}+\mathrm{G}_{\mathrm{y}}^{2}}
$$

$G_{x}$ and $G_{y}$ are the first derivative operators as given in (7) and (8).

$$
\begin{aligned}
& \mathrm{G}_{\mathrm{x}}=[f(x+1, y-1)+2 f(x+1, y)+f(x+1, y+1)] \\
& -[f(x-1, y-1)+2 f(x-1, y)+f(x-1, y+1)]
\end{aligned}
$$

And,

$$
\begin{aligned}
& \left.\mathrm{G}_{\mathrm{y}}=[f(x+1, y-1))+2 f(x, y+1)+f(x+1, y+1)\right] \\
& -\left[f(x-1, y-1)+2 f\left(x_{y} y-1\right)+f(x-1, y+1)\right]
\end{aligned}
$$

\section{G. OTSU thresholding}

It is used to perform automatic image thresholding and to obtain a binary vein structure. After thresholding, the LOG filtered image is converted into a binary image. The binary image thus obtained comprises all the features which we required in the classification of the D-CNN model. The OTSU is calculated as

$$
\sigma_{w}^{2}=W_{b} \sigma_{b}^{2}+W_{f} \sigma_{f}^{2}
$$

Where, $W_{b}=$ weight of background, $W_{f}=$ weight of foreground, $\sigma_{b}^{2}=$ variance of background, $\sigma_{f}^{2}=$ variance of foreground 


\section{Proposed Method: Detection of DR Using MRHE-FEED AND CLASSIFICATION USING D-CNN}

The main goal of our work is to develop a more efficient and less complex algorithm for medical image enhancement, segmentation and classification. In order to achieve this we have identified that a lot of image processing techniques involve more number of sub-processes, which eventually increases execution time and complexity.

In this proposed paper, we have developed a framework for detection and classification of DR. We have used the Mathematical Morphological and other image enhancement technique to extract the features of DR such as Retinal vessels, MA's, Exudates, and haemorrhages. The classification is done relying upon the area of Retinal vessels, area of MA's, area of exudates, and area of haemorrhages using the DCNN classifier. Unlike the previous techniques mentioned in [7]- [12], they have used different stages in pre-processing and feature extraction. To reduce the overall execution time, complexity to train the network and to increase the accuracy and recall, we have proposed a pre-processing and feature extraction technique that can be executed in a single stage.

Initially, to perform MRHE segmentation, we have considered only the green channel of an RGB fundus image to extract the features to detect DR as a part of pre-processing. Because the input which we consider often suffers from low contrast, high noise, and different levels of brightness. Then, we have performed Morphological operations on the fundus eye images to remove the imperfections in the structure of the image. Later, the contrast enhancement of the output image is obtained using the CLAHE and Sobel edge detection approach. To reduce the noise, we have applied local thresholding, Gaussian blurring, Laplacian of Gaussian, and OTSU thresholding techniques. The general flowchart of the proposed method is as shown in Fig. 2.

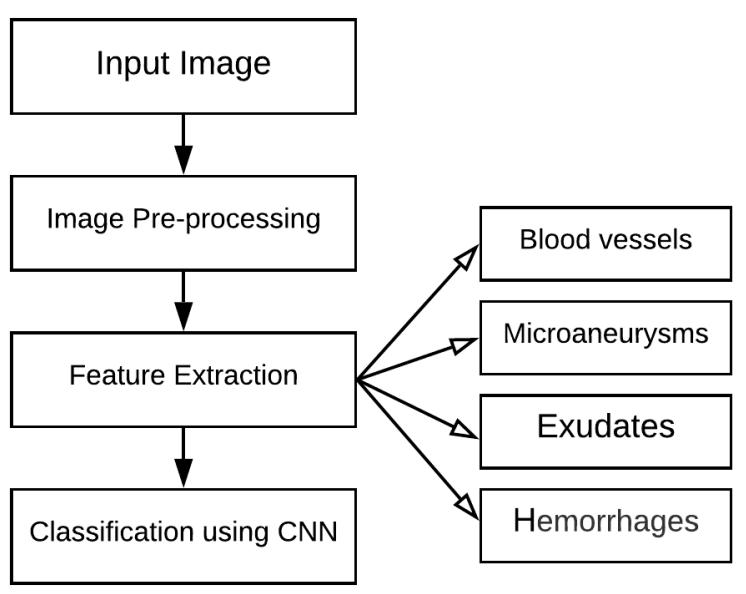

Fig. 2. Flowchart of proposed method

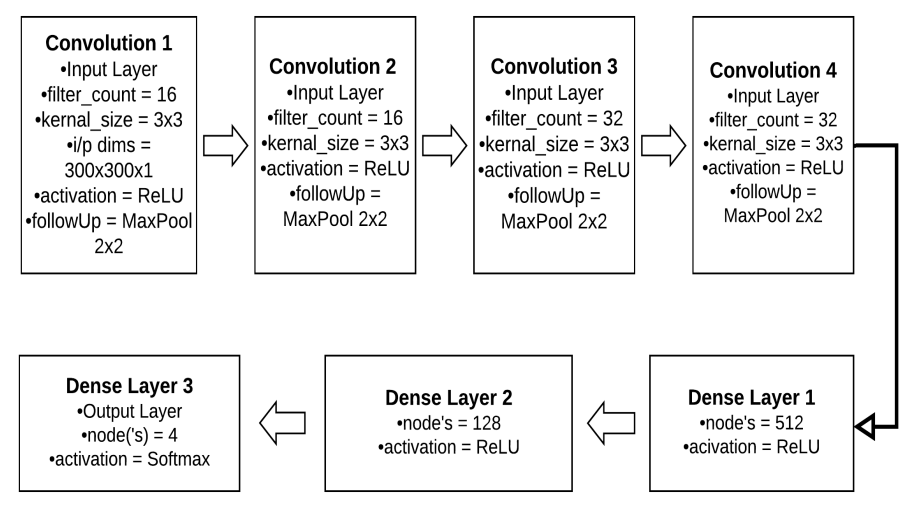

Fig. 3. Layers description of D-CNN model

A. Pre-processing and Feature Extraction: MA's, Retinal veins, Haemorrhages, Exudates segmentation and detection

In the proposed work, a hybrid pre-processing and feature extraction technique, namely MRHE using FEED was introduced to enhance all the features of DR in a precise manner with less complexity. The principal ideology of the proposed technique is to enhance the features like retinal veins, MA's, haemorrhages, and exudates detection; simultaneously rescind the other features. Our main objective is to accomplish all the pre-processing and feature extraction in a solitary go.

\section{B. Classification using D-CNN}

As we have extracted all the features related to by the proposed MHRE-FEED technique, we have opted D-CNN as a classifier to evaluate the performance of the proposed technique based on the accuracy and recall, detailed architecture of our model is explained below.

The architecture of D-CNN for the proposed method is as follows. It comprises of 4 convolution layers and 4 maxpooling layers stacked on each other alternatively. Each of the 4 convolution layers has $16,16,32$, and 64 filters respectively with a common kernel of size $3 \times 3$. All the 4 max-pooling layers have a window of size $2 \times 2$. The fully connected layer has 2 hidden layers with 512 and 128 nodes respectively and one output layer. The output layer has 4 classes which represent Normal, Background DR, Proliferate DR, and MA's. All the layers and functions used in D-CNN model are shown in Fig. 3.

\section{EXPERIMENTAL RESULTS}

\section{A. Data Description}

The dataset we have used is taken from the STructured Analysis of the REtina (STARE) Database [12], which comprises 400 retinal images taken under various imaging conditions using fundus photography and these are categorized into 13 sub-categories. From those, we had chosen only 4 categories (retinal veins, MA's, exudates, and haemorrhages). Initially, we have taken only 77 fundus images; after using image augmentation techniques to increase our data set size, 


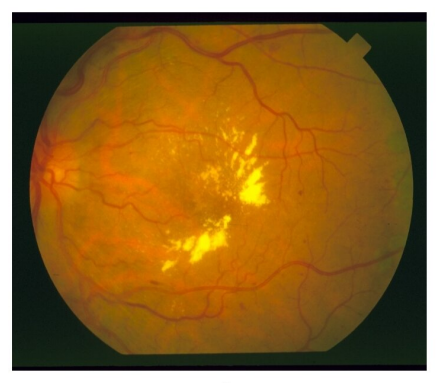

a)

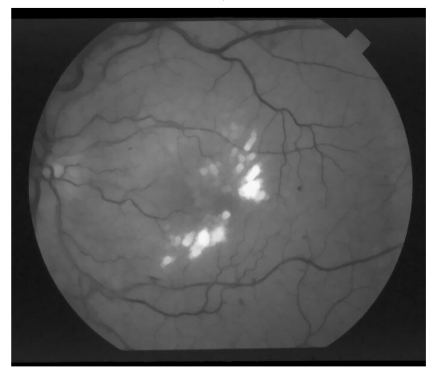

c)

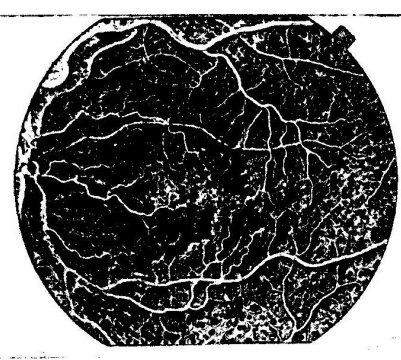

e)

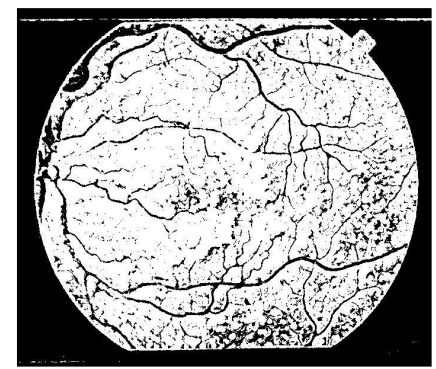

g)

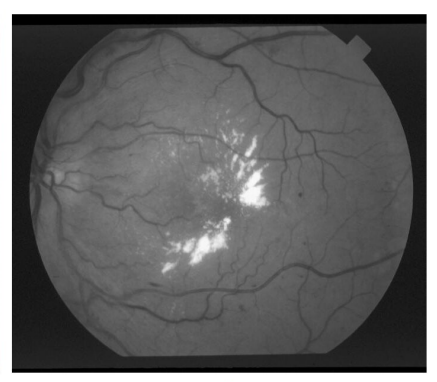

b)

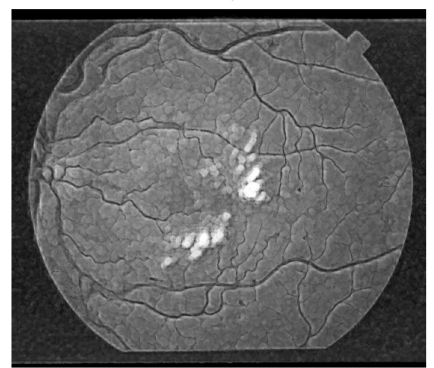

d)

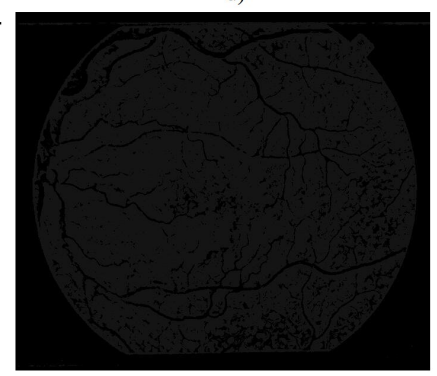

f)

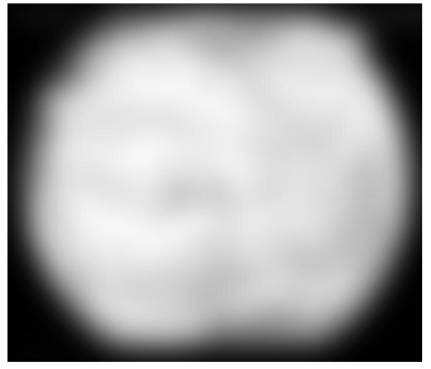

h)

Fig. 4. Proposed FEED output: (a) Raw image, (b) Green channel image, (c) Morphological opening, (d) CLAHE output, (e) Local thresholding, (f) Minimums between (b) and (e), (g) Applied CLAHE on (f), (h) Gaussian blur

we have 4928 fundus images in total while training and testing the D-CNN network. From the dataset; we have used $80 \%$ (i.e. 3943 images) of the data for training and $20 \%$ (i.e. 985 images) of the data for validating and testing.

\section{B. Pre-processing and Feature Extraction: Retinal veins seg- mentation, MAs detection, and Exudates detection}

To illustrate our proposed technique for feature extraction, we had chosen a fundus image, as shown in Fig. 4(a). Only the green channel from the RGB fundus image is extracted, as

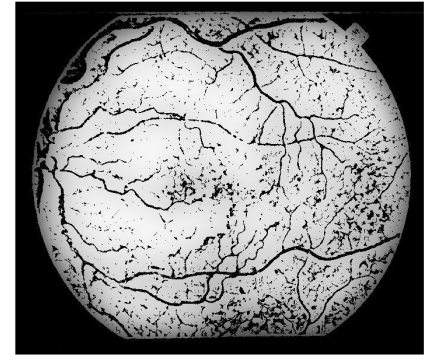

i)

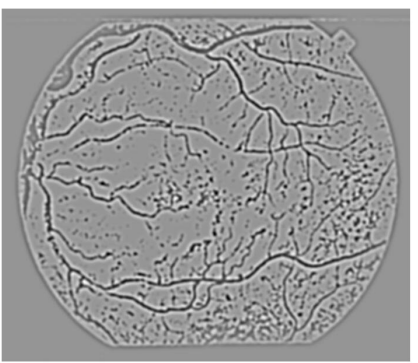

k)

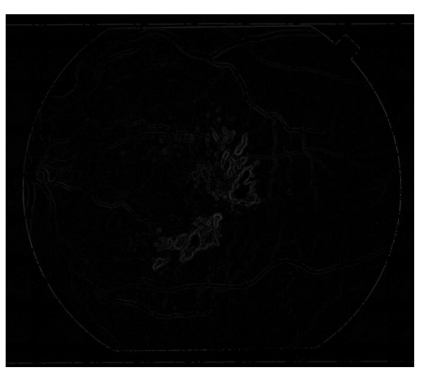

m)

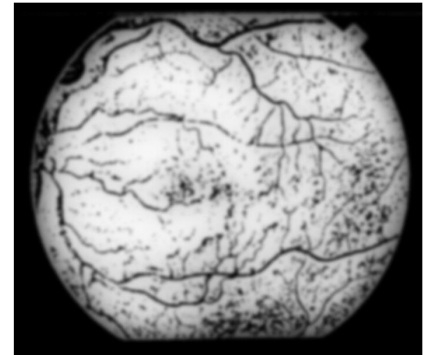

j)

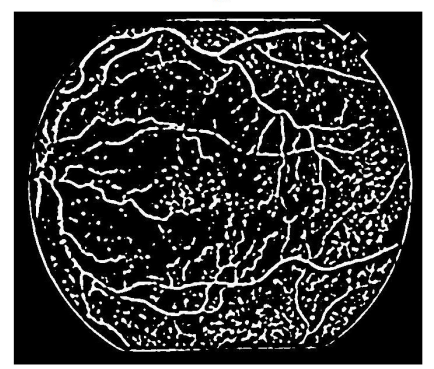

1)

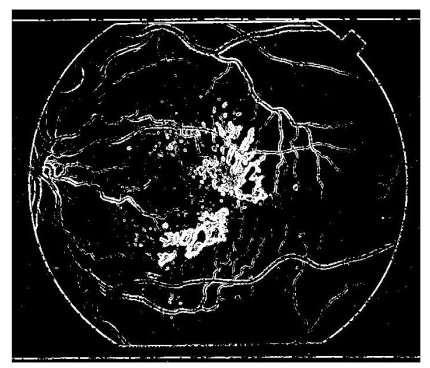

n)
Fig. 5. Proposed FEED output: (i) Minimums between (g) and (h), (j) Gaussian blur, (k) Applied Laplacian of Gaussian (LOG), (l) Applied OTSU thresholding, (m) Applied Sobel edge detector, (n) Desired output of feature extraction after applying OTSU thresholding on (m)

shown in Fig. 4(b). Then, we had applied the morphological opening with a disk of radius $=4$; the result obtained is shown in Fig. 4(c). Now, we have applied CLAHE with a kernel of size 5 on Fig. 4(c) to enhance the presence of required features from the image, as shown in Fig. 4(d). Local thresholding is computed in Fig. 4(d), and the resultant image in Fig. 4(e). A minimum is taken in between Fig. 4(b) and Fig. 4(e) to enhance the edges in comparison with the background of the image as shown in Fig. 4(f). Again, we have applied CLAHE on Fig. 4(f) for enhancing the required feature as mentioned above; the resultant image is shown in Fig. 4(g). This output was convolved with a Gaussian blurring to illuminate the pattern with a kernel of $\sigma=25$, as shown in Fig. 4(h).

Later, minimum is taken between Fig. 4(g) and Fig. 4(h) to enhance the edges in comparison with the background of the images, as shown in Fig. 5(i). The output was again convolved with a Gaussian blurring kernel of $\sigma=3$ as shown in Fig. $5(\mathrm{j})$, which helps to develop continuity in the retinal veins structure. To eliminate the non-uniform background problem, we convolved the image obtained in Fig. 5(j) with a LOG 
kernel of size 3 as shown in Fig. 5(k). OTSU thresholding was computed on Fig. 5(k), and the resultant is shown in Fig. 5(1). Later, Sobel edge detection is applied with a masking layer as in Fig. 5(g) and the resultant image is shown in Fig. 5(m). Finally, OTSU thresholding is applied on Fig. 5(m) to obtain a better version of the fundus image with all the features (retinal vessels, MA's, exudates, and haemorrhages) popped out and thus obtained output is shown in Fig. 5(n).

\section{Classification using D-CNN}

$\mathrm{CNN}$ architecture comprises three layers, namely Convolutional Layer (CL), Max Pooling Layer (MPL), and FullyConnected Layer (FCL). In the proposed D-CNN Network architecture; We have used 4 CL's of kernel size $=3$, 4 MPL's with window size $=[2,2]$ and 3 FCL's with 512,128 and 4 nodes respectively.

$$
\operatorname{ReLU} R(x)=\left\{\begin{array}{c}
x \forall x>0 \\
0 \forall x<0 \\
\text { undefined at } x=0
\end{array}\right.
$$

But for computational purposes, we take $\mathrm{R}(0)$ as 0 .

The network trained to detect salient features such as retinal veins, MA's, exudates along with haemorrhages. Convolution is performed between the filter (kernel). The obtained outcome is passed through a non-linear activation function referred to as a rectified linear unit (ReLU) as given mathematically in (10). Later, the obtained image is passed to the MPL, to downsample the image for accelerating the training process and to reduce the amount of memory consumed by the network.

Initially, we have resized the images to $300 \times 300$ and normalized them. Later, we apply CL to enhance the features of the image. The first two CL's comprises of 16 filters, and last two CL's comprises of 32 filters each. The image is not down-sampled after the application of CL but is done with the application of MPL. In the MPL, the maximum value within the window is pooled into an output matrix. We have stacked these 4CL's and 4MPL's alternatively starting with CL. The output of the final MPL comprises of 32 images with each image of dimensions $=18 \times 18$.

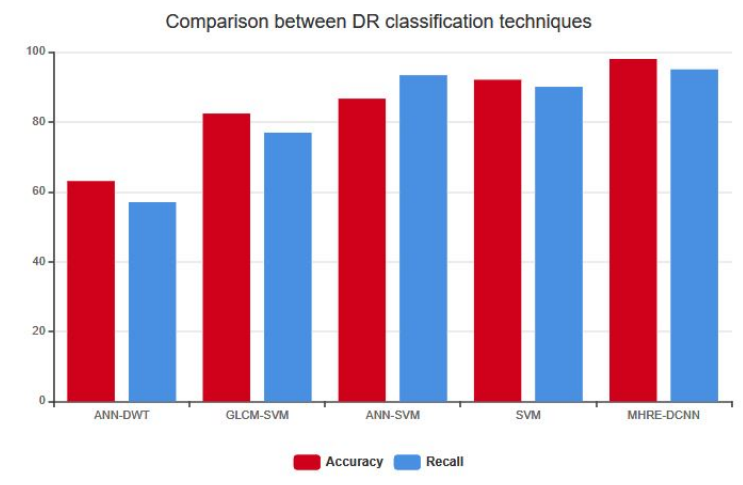

Fig. 6. Histogram comparing accuracies of existing DR classification techniques with the proposed technique
Now, ReLU is applied to the output of every FCL. We have trained the classifier until the desired amount of accuracy and recall was achieved. Over-training leads to over-fitting. Overfitting happens when a model learns the detail and noise in the training data to the extent that it negatively impacts the performance of the model on new data. At last, the final output thus obtained with FCL is mapped by the non-linear softMax activation function.

\section{DISCUSSION}

An automated framework was developed to identify DR or non-DR from the given retinal fundus image; in which various algorithms were used to automatically detect features such as retinal veins, MA's, exudates, and haemorrhages and henceforth causes ophthalmologists to identify and detect DR and forestalls the vision loss. The algorithm achieved a high percentage of sensitivity and specificity for detection of DR. The Comparison of training parameters such as Accuracy, Recall, and Precision is shown in Fig. 7. Moreover, The Comparison between different DR classification techniques with the proposed technique is shown in Fig. 6.

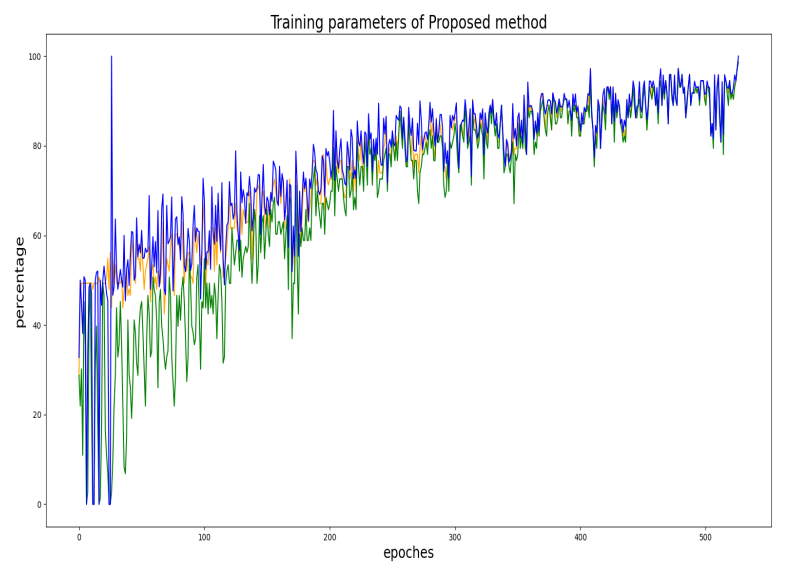

Fig. 7. Comparison of training parameters: Accuracy (orange), Recall (green), Precision (blue)

The proposed framework is more compelling and proficient than different frameworks, considered in previous works, to show the signs of improvement comprehension of the exhibition of our model we have compared with the previous work of others, for instance, with [8]- [11]. In [8], the accuracy for the best case is seen as $92 \%$ while the accuracy for the current technique was $98 \%$. In [9], the best case recall is recognized to be $93.3 \%$ though the recall determined for the current method is $95 \%$. As compared by the information given above the current technique seems, by all accounts, to be giving more accurate output. The parameters used for evaluating the classifiers in this study are sensitivity, and accuracy as can be calculated by equation (11) to (13).

$$
\text { Sensitivity }=\frac{\mathrm{TP}}{\mathrm{TP}+\mathrm{FN}}
$$




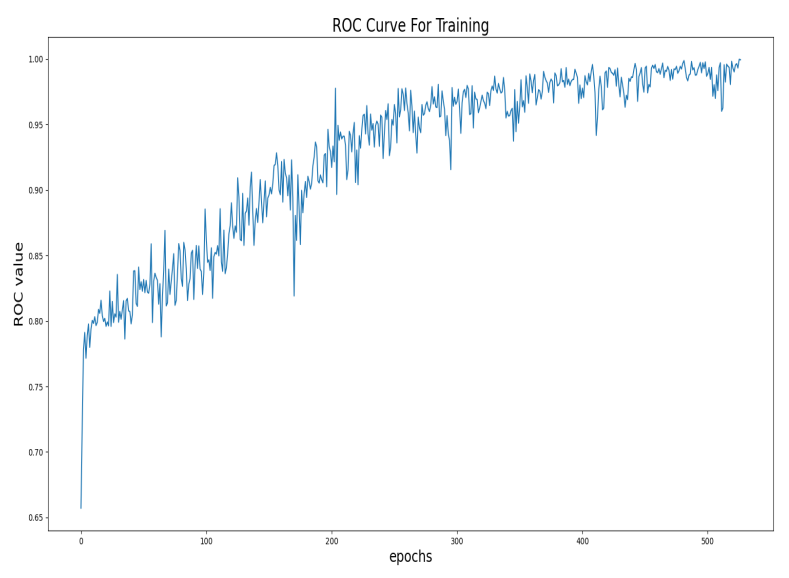

Fig. 8. ROC curve of training data

$$
\text { Accuracy }=\frac{\mathrm{TP}+\mathrm{TN}}{\mathrm{TP}+\mathrm{FP}+\mathrm{TN}+\mathrm{FN}}
$$

where, $\mathrm{TP}=$ True Positive, $\mathrm{TN}=$ True Negative, FN=False Negative, FP=False Positive

The ROC curve which is plotted with TPR against FPR is shown in Fig. 8 .

TABLE I

COMPARISON OF ACCURACY AND RECALL BETWEEN VARIOUS CLASSIFICATION TECHNIQUES AND PROPOSED MRHE-DCNN TECHNIQUE.

\begin{tabular}{|l|l|l|}
\hline \multirow{2}{*}{$\begin{array}{l}\text { Classification } \\
\text { techniques }\end{array}$} & \multicolumn{2}{|c|}{ DR CLASSIFICATION } \\
\cline { 2 - 3 } SVM [8] & Accuracy (\%) & Recall $(\%)$ \\
\hline ANN-SVM [9] & 92 & 90 \\
\hline ANN-DWT [10] & 86.6 & 93.3 \\
\hline GLCM-SVM [11] & 63 & 57 \\
\hline MHRE-DCNN & 82.35 & 76.92 \\
\hline
\end{tabular}

\section{CONCLUSION}

This paper presents an hybrid image processing method for the detection of DR using MRHE-FEED and classification using D-CNN. As a part of pre-processing and feature extraction we have proposed FEED technique to enhance and detect MA's, retinal veins, haemorrhages, and exudates from the retinal fundus images. The obtained images are then classified by using the D-CNN classifier. The classification is performed based upon values of blood vessel area, MA's area, haemorrhages, and exudates area. The proposed method achieved $95.33 \%$ sensitivity and $98.63 \%$ accuracy, which is then compared with the existing techniques as mentioned in Table I. In each and every aspect the proposed method outperformed the existing techniques. In future, our work can be further extended to detect and classify the different levels of DR in retinal fundus images.

\section{ACKNOWLEDGMENT}

All the authors of this paper acknowledge Department of Electronics and Electrical Engineering of Indian Institute of Technology Guwahati for providing the summer internship.

\section{REFERENCES}

[1] A. U. Naik and R. K. Kulkarni, "Artificial Neural Network-based Detection of Diabetes and its Effects on Vision - A survey," 2020 5th International Conference on Communication and Electronics Systems (ICCES), COIMBATORE, India, 2020, pp. 1113-1118, doi: 10.1109/ICCES48766.2020.9138057.

[2] S. Wang et al., "Localizing MA's in Fundus Images Through Singular Spectrum Analysis," in IEEE Transactions on Biomedical Engineering, vol. 64, no. 5, pp. 990-1002, May 2017, doi: 10.1109/TBME.2016.2585344.

[3] M. S. Ahmed and B. Indira, "Morphological technique for detection of MA's from RGB fundus images," 2017 International Conference on Wireless Communications, Signal Processing and Networking (WiSPNET), Chennai, 2017, pp. 44-47, doi: 10.1109/WiSPNET.2017.8299716.

[4] S. Kumar and B. Kumar, "Diabetic Retinopathy Detection by Extracting Area and Number of MA from Colour Fundus Image," 2018 5th International Conference on Signal Processing and Integrated Networks (SPIN), Noida, 2018, pp. 359-364, doi: 10.1109/SPIN.2018.8474264.

[5] S. B. Patil and B. P. Patil, "Automatic Detection of MA's in Retinal Fundus Images using Modified High Boost Filtering, Line Detectors and OC-SVM," 2020 International Conference on Industry 4.0 Technology (I4Tech), Pune, India, 2020, pp. 148-153, doi: 10.1109/I4Tech48345.2020.9102677.

[6] Harini $\mathrm{R}$ and Sheela N, "Feature extraction and classification of retinal images for automated detection of Diabetic Retinopathy," 2016 Second International Conference on Cognitive Computing and Information Processing (CCIP), Mysore, 2016, pp. 1-4, doi: 10.1109/CCIP.2016.7802862.

[7] P. Dhal and C. Azad, "A novel approach for blood vessel segmentation with exudate detection in diabetic retinopathy," 2020 International Conference on Artificial Intelligence and Signal Processing (AISP), Amaravati, India, 2020, pp. 1-6, doi: 10.1109/AISP48273.2020.9073012.

[8] F. Cisneros-Guzmán, S. Tovar-Arriaga, C. Pedraza and A. GonzálezGutierrez, "Classification of diabetic retinopathy based on hard exudates patterns, using images processing and SVM," 2019 IEEE Colombian Conference on Applications in Computational Intelligence (ColCACI), Barranquilla, Colombia, 2019, pp. 1-5, doi: 10.1109/ColCACI.2019.8781802.

[9] C. M. Patil and Y. K. S., "An Approach of Abnormality Detection for Diabetic Retinopathy using ANN SVM," 2017 International Conference on Current Trends in Computer, Electrical, Electronics and Communication (CTCEEC), Mysore, 2017, pp. 323-327, doi: 10.1109/CTCEEC.2017.8455152

[10] N. Kashyap, D. K. Singh and G. K. Singh, "Mobile phone based diabetic retinopathy detection system using ANN-DWT," 2017 4th IEEE Uttar Pradesh Section International Conference on Electrical, Computer and Electronics (UPCON), Mathura, 2017, pp. 463-467, doi: 10.1109/UPCON.2017.8251092.

[11] A. Z. Foeady, D. C. R. Novitasari, A. H. Asyhar and M. Firmansjah, "Automated Diagnosis System of Diabetic Retinopathy Using GLCM Method and SVM Classifier," 2018 5th International Conference on Electrical Engineering, Computer Science and Informatics (EECSI), Malang, Indonesia, 2018, pp. 154-160, doi: 10.1109/EECSI.2018.8752726

[12] http://cecas.clemson.edu/ ahoover/stare/ 